\title{
Radiative Energy Loss in a Finite Dynamical QCD Medium
}

\author{
Magdalena Djordjevic ${ }^{1}$ and Ulrich Heinz ${ }^{1,2}$ \\ ${ }^{1}$ Physics Department, The Ohio State University, Columbus, Ohio 43210, USA \\ ${ }^{2}$ CERN, Physics Department, Theory Division, CH-1211 Geneva 23, Switzerland
}

(Received 17 February 2008; published 10 July 2008)

\begin{abstract}
The radiative energy loss of a quark jet traversing a finite size QCD medium with dynamical constituents is calculated to first order in opacity. Although finite size corrections reduce the energy loss relative to an infinite dynamical QCD medium, under realistic conditions it remains significantly larger than in a static medium. Quantitative predictions of jet suppression in relativistic heavy ion collisions must therefore account for the dynamics of the medium's constituents. Finite size effects are shown to induce a nonlinear path length dependence of the energy loss. Our results suggest a simple general mapping between energy loss expressions for static and dynamical QCD media.
\end{abstract}

PACS numbers: 25.75.Nq, 12.38.Mh, 12.38.Qk

Studying the suppression of high transverse momentum hadrons is a powerful tool to map out the density of a QCD plasma created in ultrarelativistic heavy ion collisions $[1,2]$. Since this suppression (called jet quenching) results from energy loss of fast partons moving through the plasma [3-6], quantitative jet quenching predictions require reliable energy loss calculations.

In the majority of currently available studies the medium-induced radiative energy loss is computed by assuming that the QCD medium consists of randomly distributed static scattering centers ("static QCD medium"). We recently calculated [7], at leading order in opacity, the heavy quark radiative energy loss in an infinite QCD medium consisting of dynamical constituents and found that the energy loss increases by almost a factor 2 relative to an equally dense static medium. However, this calculation was performed in the Bethe-Heitler limit which is well known [8] to overpredict radiative energy loss since it does not include coherence and finite size effects. As the medium created in heavy ion collisions has finite size, it is essential to explore how the qualitative conclusions obtained in [7] change once such effects are included. We find that finite size and coherence effects decrease the radiative energy loss per unit path length more strongly in a dynamical than in a static medium, reducing the energy loss ratio between equally dense dynamical and static media. Still, the ratio remains significantly larger than unity even if the medium is finite, showing that for quantitative predictions of radiative energy loss it is important to account for the dynamic nature of the QCD medium's constituents.

We briefly outline the computation of the mediuminduced radiative energy loss for a heavy quark to first order in opacity. We consider a QCD medium of size $L$ and assume that the heavy quark is produced at time $x_{0}=0$ at the left edge of the medium, traveling right. Collisions with partons in the medium induce the radiation of gluons, causing the quark to lose energy. The radiative energy loss rate can be expanded in the number of scattering events suffered by the heavy quark. This is equivalent to an expansion in powers of the opacity. For a finite medium, the opacity is given by the product of the medium density with the transport cross section, integrated along the path of the heavy quark. The leading (first order) contribution corresponds to one collisional interaction with the medium, accompanied by the emission of a single gluon. This is the process we compute.

To introduce the finite size of the medium we start from the approach described in [9] and follow the procedure used in [10]. The medium extends for a length $L$ from the production point of the energetic heavy quark, and the collisional interaction inducing the radiation of a gluon occurs after a distance $l<L$ inside the medium.

As in [7], we describe the medium by a thermalized quark-gluon plasma at temperature $T$ and zero baryon density, with $n_{f}$ effective massless quark flavors in equilibrium with the gluons. Three typical Feynman diagrams contributing to the radiative quark energy loss at first order in opacity are shown in Fig. 1. The diagrams are evaluated in finite temperature field theory [11,12], using Hard Thermal Loop (HTL) resummed propagators [12] for all gluons. A full account of the calculation will be presented elsewhere [13]. A flavor of what it involves is given by Fig. 1. The elliptic blob represents a source $J$ which at time $x_{0}$ produces an energetic quark with momentum $p^{\prime}$. In diagram 1(a) the produced quark is on-shell. It first radiates a gluon with momentum $k=\left(\omega, k_{z}, \boldsymbol{k}\right)$ and then exchanges a virtual gluon of momentum $q=\left(q_{0}, q_{z}, \boldsymbol{q}\right)$ with a parton in the medium, finally emerging (at the dashed line denoting an on-shell cut through the amplitude represented by the diagram) with (measured) momentum $p=\left(E, p_{z}, \boldsymbol{p}\right)$ [14]. Since the energetic quark produced by the source $J$ can be off-shell, we also have contributions such as those in Figs. 1(b) and 1(c). Amplitudes 1(b) and 1(c) interfere with amplitude 1(a), leading to the appearance of LPM-like effects once all relevant contributions are summed. The present calculation differs from that in [8] by the use of HTL gluon propagators to describe the interaction of the quark with the medium, and from that in Ref. [7] by allowing the jet to be on- or off-shell and restricting the vertices corresponding to gluon exchange to be located 


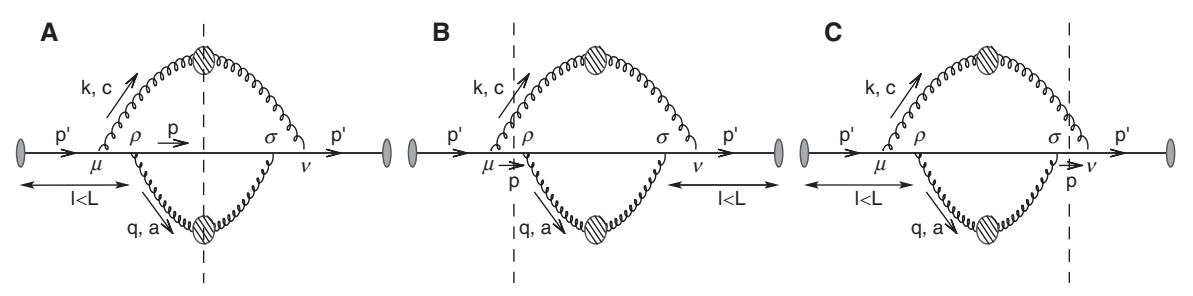

FIG. 1. Three typical Feynman diagrams contributing to the quark radiative energy loss in a finite size dynamical QCD medium at first order in opacity. See text for discussion. inside the medium, i.e., at $l<L$ [15]. We use the same kinematic approximations as in [7,8]; accordingly, the gluon propagators for exchanged gluons in Fig. 1 contribute only for spacelike momenta $\left(q_{0}<|\overrightarrow{\mathbf{q}}|\right)$ and those for radiated gluons only for timelike momenta $(\omega>|\overrightarrow{\mathbf{k}}|)$ $[7,10]$. We also assume that $J$ changes slowly, i.e., $J\left(p^{\prime}\right) \approx$ $J(p)[16]$.

Explicit calculation of all 21 diagrams contributing to first order in the opacity [13] yields the following expression for the fractional radiative energy loss $(\mu=$ $g T \sqrt{N_{c} / 3+N_{f} / 6}$ is the Debye screening mass and parametrizes the density of the medium):

$$
\begin{aligned}
\frac{\Delta E_{\mathrm{dyn}}}{E}= & \frac{C_{R} \alpha_{s}}{\pi} \frac{L}{\lambda_{\mathrm{dyn}}} \int d x \frac{d^{2} k}{\pi} \frac{d^{2} q}{\pi} \frac{\mu^{2}}{\boldsymbol{q}^{2}\left(\boldsymbol{q}^{2}+\mu^{2}\right)} \\
& \times\left(1-\frac{\sin \left(\frac{(\boldsymbol{k}+\boldsymbol{q})^{2}+\chi}{x E^{+}} L\right)}{\frac{(\boldsymbol{k}+\boldsymbol{q})^{2}+\chi}{x E^{+}}} L\right. \\
& \times \frac{2(\boldsymbol{k}+\boldsymbol{q})}{(\boldsymbol{k}+\boldsymbol{q})^{2}+\chi}\left(\frac{(\boldsymbol{k}+\boldsymbol{q})}{(\boldsymbol{k}+\boldsymbol{q})^{2}+\chi}-\frac{\boldsymbol{k}}{\boldsymbol{k}^{2}+\chi}\right) .
\end{aligned}
$$

Here $\lambda_{\text {dyn }}^{-1} \equiv C_{2}(G) \alpha_{s} T=3 \alpha_{s} T$ defines the "dynamical mean free path" [7], $\alpha_{s}=\frac{g^{2}}{4 \pi}$ is the strong coupling constant, and $C_{R}=\frac{4}{3}$. Further, $\chi \equiv M^{2} x^{2}+m_{g}^{2}$ where $x$ is the longitudinal momentum fraction of the heavy quark carried away by the emitted gluon and $m_{g}=\frac{\mu}{\sqrt{2}}$ is the effective mass for gluons with hard momenta $k \gtrsim T$.

Similar to the infinite medium studied in [7], each individual diagram contributing to the energy loss in a finite dynamical medium diverges logarithmically in the limit of zero transverse momentum exchange $\boldsymbol{q} \rightarrow 0$, [13]. In a dynamical QCD medium both transverse and longitudinal gluon exchange contribute to radiative energy loss [17]; while Debye screening renders the longitudinal gluon exchange infrared finite, transverse gluon exchange causes a logarithmic singularity due to the absence of magnetic screening [12]. Remarkably, this singularity is found to cancel in the sum over all diagrams [13], naturally regulating the energy loss rate.

We can compare the radiative energy loss rate in a dynamical medium (1) to the analogous result for a static medium. One can rewrite the DGLV expression [8] for the first order radiative energy loss in a static QGP, $\Delta E_{\text {stat }} / E$, in the same form as Eq. (1), except for two simple substitutions: (1) $\lambda_{\text {dyn }}$ is replaced by the "static mean free path" $\lambda_{\text {stat }}$, defined by $[7,18]$

$$
\frac{1}{\lambda_{\text {stat }}}=\frac{1}{\lambda_{g}}+\frac{1}{\lambda_{q}}=6 \frac{1.202}{\pi^{2}} \frac{1+\frac{n_{f}}{4}}{1+\frac{n_{f}}{6}} 3 \alpha_{s} T=c\left(n_{f}\right) \frac{1}{\lambda_{\mathrm{dyn}}},
$$

where $c\left(n_{f}\right) \equiv 6 \frac{1.202}{\pi^{2}} \frac{1+n_{f} / 4}{1+n_{f} / 6}$ is a slowly increasing function

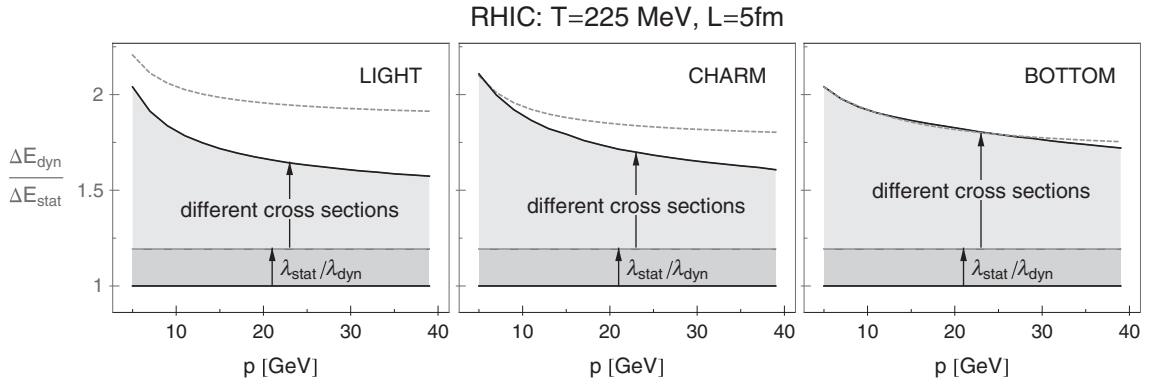

LHC: $\mathrm{T}=400 \mathrm{MeV}, \mathrm{L}=5 \mathrm{fm}$

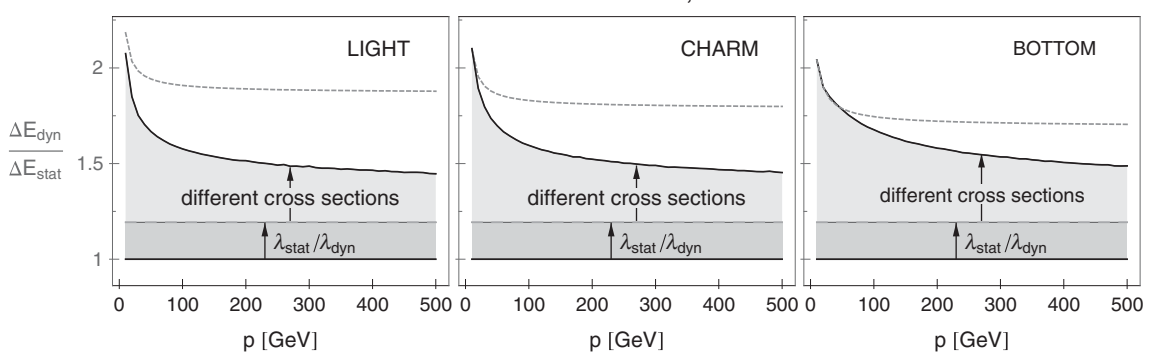

FIG. 2. Ratio of the radiative energy loss in finite dynamical and static QCD media of length $L=5 \mathrm{fm}$ for light, charm and bottom quarks (left, center, and right panels, respectively), as a function of initial quark momentum $p$. Top row: RHIC conditions (average medium temperature $T=225 \mathrm{MeV}$ ). Bottom row: LHC conditions $(T=400 \mathrm{MeV})$. The dashed curves show the corresponding energy loss ratio in an infinite QCD medium for comparison. 
of $n_{f}$ that varies between $c(0) \approx 0.73$ and $c(\infty) \approx 1.09$. For $n_{f}=2.5$ (see below) $c(2.5) \approx 0.84$. (2) The effective cross section under the integral (1) for the energy loss rate is replaced as

$$
\left[\frac{\mu^{2}}{\boldsymbol{q}^{2}\left(\boldsymbol{q}^{2}+\mu^{2}\right)}\right]_{\mathrm{dyn}} \mapsto\left[\frac{\mu^{2}}{\left(\boldsymbol{q}^{2}+\mu^{2}\right)^{2}}\right]_{\mathrm{stat}} .
$$

Taken together, these differences will be seen to cause a significant increase of the heavy quark energy loss rate in dynamical compared to static QCD media.

These two simple replacements are identical to those found in the Bethe-Heitler limit [7]. The simplicity of this substitution rule is surprising, given the complexity of the calculations and their different structure for static [8] and dynamical [13] media. (Remember the infrared divergences in the dynamical case which cancel only after summing all 21 diagrams but do not arise at all in the static case.) The integrands in Eq. (1) and its static analog are significantly different from the corresponding ones in the Bethe-Heitler limit [8], giving rise (as we will see) to a different energy dependence of the dynamic/static energy loss ratio. Nonetheless the same simple substitution rule is found to apply, suggesting a possibly general mapping between static and dynamic QCD media.

The study presented here considers a finite, optically thin dynamical QCD medium (QGP), extending the DGLV approach [8] to include parton recoil. In this sense it is complementary to the work by Arnold, Moore and Yaffe [19] who study energy loss in an infinite, optically thick QGP. We note that the AMY approach [19] yields the same form (3) for the effective cross section in a dynamical QCD medium as found here (see also [20]), supporting our conjecture above.

We now highlight finite size effects in a dynamical QCD medium, to first order in opacity, with a few numerical results for radiative energy loss. In Fig. 2 we show the ratio of the radiative energy loss rates in equally dense dynamical and static QCD media as a function of the initial energy of the fast quark, under RHIC and LHC conditions. In both cases a medium of length $L=5 \mathrm{fm}$, a constant value of $\alpha_{s}=0.3$, and a chemically equilibrated QGP with $n_{f}=$
2.5 effective light quark flavors is assumed. The light quark mass is assumed to be dominated by the thermal mass, $M_{q}=\mu / \sqrt{6}$, where $\mu$ is the Debye screening mass. For the charm and bottom masses we use $M_{c}=1.2 \mathrm{GeV}$ and $M_{b}=4.75 \mathrm{GeV}$, respectively. For $\mathrm{Au}+\mathrm{Au}$ collisions at top RHIC energies we assume an average medium temperature of $T=225 \mathrm{MeV}$, for $\mathrm{Pb}+\mathrm{Pb}$ at the $\mathrm{LHC}$ we take $T=400 \mathrm{MeV}$.

In all cases, the energy loss is seen to be significantly larger in the dynamical than in the static medium. A common factor to all situations (large and small jet quark masses, hotter and cooler media, finite and infinite media) is the $\mathcal{O}(20 \%)$ increase of the energy loss in dynamical media arising from the shorter mean free path $\lambda_{\mathrm{dyn}} \approx$ $0.84 \lambda_{\text {stat }}$. The additional increase arising from the change (3) in cross section is larger; in the energy range shown in Fig. 2 it ranges from about $25 \%$ to over $100 \%$, depending on medium temperature and the mass and energy of the fast quark. The reduction of the energy loss ratio $\frac{\Delta E_{\text {dyn }}}{\Delta E_{\text {stat }}}$ by finite size corrections is seen to be larger for lighter quarks and larger jet energies. The smallest finite size corrections and, in the end, the biggest dynamical increase are seen for bottom quarks at RHIC.

Furthermore, in [7] we found that dynamical medium effects are largest for light quarks and decrease with quark mass. Here we see the opposite tendency: after finite size correction the energy loss ratio $\frac{\Delta E_{\mathrm{dyn}}}{\Delta E_{\mathrm{stat}}}$ becomes smallest for light quarks, increasing with quark mass. Such behavior is important, since it may contribute toward understanding the observed large suppression of nonphotonic electrons in central $\mathrm{Au}+\mathrm{Au}$ collisions at RHIC [21].

Figure 3 shows that the strength of the finite size corrections correlates with the dependence of the fractional energy loss on the thickness $L$ of the medium. In the BetheHeitler limit studied for infinite media in Ref. [7], quarks of all masses and energies lose energy at fixed rate $\frac{\Delta E}{\Delta z}$, resulting in a linear dependence of the radiative energy loss on the length $L$ traveled by the quark. In contrast, Fig. 3 shows a nonlinear $L$-dependence that becomes perfectly quadratic (corresponding to the deep Landau-
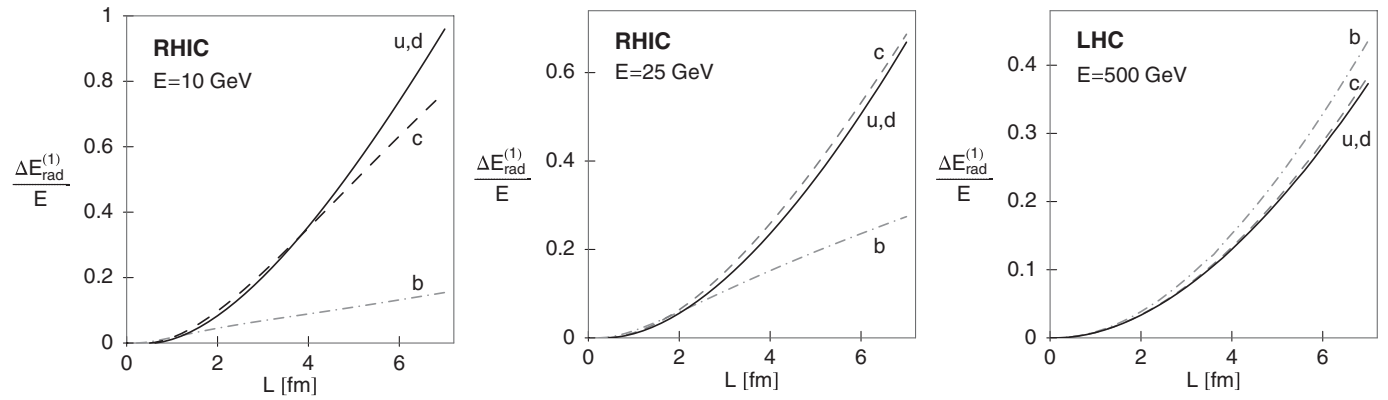

FIG. 3. First order fractional radiative energy loss as a function of medium thickness $L$ for initial jet energies $E=10,25$, and $500 \mathrm{GeV}$ (left, center, and right panels, respectively). The two left panels correspond to RHIC conditions, the right panel to LHC conditions. Solid, dashed, and dot-dashed lines describe light, charm, and bottom quark energy loss, respectively. 
Pomeranchuk-Migdal (LPM) limit [22]) for large jet energy [see Eq. (4) below]. The weakest deviations from the linear Bethe-Heitler $L$-dependence are seen for low-energy bottom quarks at RHIC, where Fig. 2 (top row, right panel) also shows the smallest finite size correction. The $L$ dependence is closest to quadratic for light quarks and for very energetic charm and bottom quarks at the LHC where also the finite size effects are largest. This shows that the finite size corrections implemented in the present calculation simulate the destructive effects of LPM interference in an infinite medium [4]. This behavior is expected [16] since the nuclear medium has finite dimensions that may be small compared to the jet radiation coherence length, especially in the case of light partons or high jet energies. Because of this, in finite size media the basic formation time physics developed by LPM [22] leads to strong destructive interference effects on the quark quenching, as observed in Fig. 3.

We finally point out that, contrary to the first order study in the Bethe-Heitler limit [7] where the energy loss ratio $\frac{\Delta E_{\mathrm{dyn}}}{\Delta E_{\mathrm{stat}}}$ saturates for sufficiently large quark energies, this ratio keeps decreasing with increasing quark energy once finite size corrections are accounted for. In fact, one finds analytically [13] that for asymptotically large jet energies Eq. (1) reduces to

$$
\frac{\Delta E_{\mathrm{dyn}}}{E} \approx \frac{C_{R} \alpha_{s}}{4} \frac{L^{2} \mu^{2}}{\lambda_{\mathrm{dyn}}} \ln \frac{4 E T}{\mu^{2}},
$$

and that the energy loss ratio approaches

$$
\lim _{E \rightarrow \infty} \frac{\Delta E_{\text {dyn }}}{\Delta E_{\text {stat }}}=\lim _{E \rightarrow \infty} \frac{\lambda_{\text {stat }}}{\lambda_{\text {dyn }}} \frac{\ln \frac{4 E T}{\mu^{2}}}{\ln \frac{4 E T}{\mu^{2}}-1}=\frac{\lambda_{\text {stat }}}{\lambda_{\text {dyn }}} .
$$

The static approximation thus becomes valid for asymptotically large jet energies.

In summary, we have presented a calculation to first order in opacity of the radiative energy loss of a fast quark traveling through a finite dynamical QCD medium. Finite size effects are found to be most important in the ultrarelativistic limit and they effectively reproduce the effects of destructive Landau-Pomeranchuk-Migdal interference. The calculation suggests the possibility of a general mapping between the energy loss expressions for static and dynamical media, which we conjecture to carry over to higher-order calculations. It also shows that the approximation of the medium by a random distribution of static scatterers becomes valid in the limit of asymptotically large jet energies once finite size and LPM interference effects are taken into account. For realistic jet energies and medium temperatures reachable at RHIC and LHC, however, parton recoil in the medium must be accounted for and leads to a large $(40 \%-70 \%)$ increase of radiative energy loss when compared with an equally dense static medium. This effect is largest for bottom quarks at RHIC which may be important for understanding the observed large suppression of nonphotonic electrons in central $\mathrm{Au}+$ Au collisions at RHIC [21].

Valuable discussions with Eric Braaten, Miklos Gyulassy, and Yuri Kovchegov are gratefully acknowledged. This work was supported by the U.S. Department of Energy, Grant No. DE-FG02-01ER41190.

[1] M. Gyulassy, Lect. Notes Phys. 583, 37 (2002).

[2] M. Gyulassy and M. Plümer, Nucl. Phys. A527, 641 (1991); M. Gyulassy, M. Plümer, M. Thoma, and X. N. Wang, Nucl. Phys. A538, 37 (1992); X. N. Wang and M. Gyulassy, Phys. Rev. Lett. 68, 1480 (1992).

[3] M. Gyulassy, I. Vitev, X. N. Wang, and B. W. Zhang, in Quark Gluon Plasma 3, edited by R. C. Hwa and X. N. Wang (World Scientific, Singapore, 2003), p. 123.

[4] R. Baier, Yu. L. Dokshitzer, A. J. Mueller, and D. Schiff, Phys. Rev. C 58, 1706 (1998); R. Baier, Yu. L. Dokshitzer, A. J. Mueller, S. Peigne, and D. Schiff, Nucl. Phys. B483, 291 (1997).

[5] R. Baier, D. Schiff, and B. G. Zakharov, Annu. Rev. Nucl. Part. Sci. 50, 37 (2000).

[6] A. Kovner and U. A. Wiedemann, in Quark Gluon Plasma 3, edited by R. C. Hwa and X. N. Wang (World Scientific, Singapore, 2003), p. 192.

[7] M. Djordjevic and U. Heinz, Phys. Rev. C 77, 024905 (2008).

[8] M. Djordjevic and M. Gyulassy, Phys. Lett. B 560, 37 (2003); Nucl. Phys. A733, 265 (2004).

[9] B. G. Zakharov, JETP Lett. 76, 201 (2002).

[10] M. Djordjevic, Phys. Rev. C 74, 064907 (2006).

[11] J.I. Kapusta, Finite-Temperature Field Theory (Cambridge University Press, Cambridge, England, 1989).

[12] M. Le Bellac, Thermal Field Theory (Cambridge University Press, Cambridge, England, 1996).

[13] M. Djordjevic (to be published).

[14] We use the same notation as in [7].

[15] Integrating over $l<L$ for these vertex positions leads to the $L$-dependent oscillating factors in Eq. (1) instead of the energy-conserving $\delta$-functions encountered in [7].

[16] M. Gyulassy, P. Levai, and I. Vitev, Nucl. Phys. B594, 371 (2001).

[17] X. N. Wang, Phys. Lett. B 485, 157 (2000).

[18] S. Wicks, W. Horowitz, M. Djordjevic, and M. Gyulassy, Nucl. Phys. A784, 426 (2007).

[19] P. Arnold, G. D. Moore, and L. G. Yaffe, J. High Energy Phys. 11 (2001) 057; 06 (2002) 030; 01 (2003) 030.

[20] P. Aurenche, F. Gelis, and H. Zaraket, J. High Energy Phys. 05 (2002) 043.

[21] S.S. Adler et al. (PHENIX Collaboration), Phys. Rev. Lett. 96, 032301 (2006); B. I. Abelev et al. (STAR Collaboration), Phys. Rev. Lett. 98, 192301 (2007).

[22] A. B. Migdal, Phys. Rev. 103, 1811 (1956); L. D. Landau and I. Pomeranchuk, Dokl. Akad. Nauk SSSR 92, 535 (1953). 